\title{
El trabajo precario y el trabajo decente: su impacto en la salud y en el desarrollo sostenible
}

\author{
Esperanza Vargas Jiménez ${ }^{1}$ \& Sara Paola Pérez Ramos ${ }^{2}$
}

Recibido: 11/10/2018 Aceptado: 23/10/2019

DOI: $10.21772 /$ ripo.v38n2a05

\begin{abstract}
Resumen
El trabajo decente es una propuesta y una necesidad para el combate de la precarización laboral, cada vez más acentuada en la dinámica globalizadora asociada a efectos adversos en la salud. El objetivo del presente estudio fue incursionar en el debate sobre trabajo precario y trabajo decente, evidenciando su impacto en la salud y su relevancia para el Desarrollo Sostenible, abonando al estudio de la salud fuera del campo clínico, desde una visión integradora asociándola a factores externos al ámbito individual, en este caso, al trabajo. Se concluye que la precariedad laboral es un tema de gran relevancia por sus implicaciones en la salud.Estos estudios permiten avanzar en el conocimiento de condiciones laborales inadecuadas y sus efectos, posibilitando así la implementación de políticas que supongan mejoras tanto para la salud de los trabajadores como para la competitividad de las empresas, tomando como guía y fin el Desarrollo Sostenible.
\end{abstract}

Palabras clave: trabajo precario, trabajo decente, salud, salud laboral, desarrollo sostenible.

\section{Precarious work and decent work: it's impact on health and sustainable development}

\begin{abstract}
Decent work is a proposal and a necessity for combating the precarious work, increasingly accentuated in the new globalizing dynamics associated with adverse effects on health. The aim of this study was to take part in the debate surrounding precarious work and decent work, showing their health impact and their relevance to Sustainable Development. This also contributes to the study of health outside a clinical framework, from an integrative perspective, tying it to factors outside an individual scope, such as labor, in this case. It is concluded that labor precariousness is a very relevant topic due to its health implications. These studies advance the knowledge of inadequate labor conditions and their effects, thus enabling the implementation of policies with an eye to the improvement of workers' health as well as of company competitiveness, taking Sustainable Development as guide and goal.
\end{abstract}

Key words: precarious work, decent work, health, occupational health, sustainable development.

\footnotetext{
1 Dra. en Ciencias para el Desarrollo Sustentable. Universidad de Guadalajara. Profesor docente investigador titular A.

E-mail: esperanzavgas@hotmail.com

2 Ms. en Ciencias para el Desarrollo, la Sustentabilidad y el Turismo. Universidad de Guadalajara, Centro Universitario de la Costa. Licenciada en Psicología. E-mail: paolaperam@gmail.com
}

Cómo citar este artículo: Vargas Jiménez, E.\& Pérez Ramos, S. P. (2019). El trabajo precario y el trabajo decente: su impacto en la salud y en el desarrollo sostenible. Revista Interamericana de Psicología Ocupacional, 38(2), 138-147. DOI: 10.21772/ripo.v38n2a05 


\section{Introducción}

El desarrollo sostenible, en los últimos años, ha sido abordado desde varias trincheras, teniendo como principales el medio ambiente, la economía, los aspectos sociales y la buena gobernanza (Sachs, 2015). Al respecto la Organización Internacional del trabajo (OIT), ha reforzado este abordaje, al señalar que el medio ambiente y el desarrollo social ya no deben considerarse como pilares separados del desarrollo sustentable, ya que están interrelacionados. Una aproximación integral desembocaría en un medio ambiente sustentable como una vía para el desarrollo, con mejores trabajos, inclusión social y la reducción de la pobreza (International Labour Organization, 2013).

En ese sentido, el presente trabajo pretende abordar el objetivo número ocho del desarrollo sostenible, que se refiere al trabajo decente, indicando su importancia y los efectos que puede traer a la sociedad, con énfasis en los individuos, lo que implica debatir respecto a la salud y su vínculo con el trabajo. Asimismo, se mostrará el impacto que tiene el trabajo precario, contrastando a este como una problemática urgente, con especial impacto en la salud, a la que el Trabajo Decente intenta dar respuesta. Así, el objetivo del presente estudio es incursionar en el debate sobre trabajo precario y trabajo decente, evidenciando su impacto en la salud, abonando así, al estudio de la salud fuera del campo clínico, desde una visión integradora, asociándola a factores externos al ámbito individual, en este caso, al trabajo, tomando como guía de análisis y posible camino al desarrollo sostenible. De esta manera, y coincidiendo con los aportes de la International Labour Organization (2013) y Parker (2014), el trabajo constituye una variable de alto impacto en el desarrollo sostenible. La necesidad de una aproximación integral ha sido articulada en $\mathrm{Rio}+20$ por la ONU, quien ha enfatizado que el trabajo decente es una meta central y un conductor para el desarrollo y una economía sostenible
(International Labour Organization, 2013).

La articulación de estos fenómenos de estudio: trabajo decente, trabajo precario, salud y desarrollo sostenible, buscan brindar pautas generales para una mejora en la calidad de la salud de los empleados, de las empresas y de la sociedad en general. Se pretende que esta reflexión sea un referente para los nuevos debates teóricos que se abren a raíz de las consecuencias del capitalismo, que rige la economía, la sociedad y, por ende, el mercado de trabajo.

\section{Trabajo Decente}

La Organización Internacional del Trabajo (OIT) ha elaborado un programa para la comunidad del trabajo basado en la creación de empleo, los derechos en el trabajo, la protección social y el diálogo social, teniendo a la igualdad de género como objetivo transversal. La OIT (1999) indica que es urgente entre las políticas internacionales, sobre todo a raíz de la crisis financiera y económica de 2008, proporcionar empleos de calidad asociados a la protección social y al respeto de los derechos en el trabajo, a fin de alcanzar un crecimiento económico sustentable e inclusivo y erradicar la pobreza.

El trabajo decente está centrado en cuatro objetivos estratégicos: a) la promoción y crecimiento del empleo, b) el respeto a los derechos laborales consagrados en la legislación laboral, c) la protección social contra las situaciones de vulnerabilidad y d) el fomento del diálogo social (Organización Internacional del Trabajo, 1999)1999.

La OIT señala que el trabajo decente está vinculado a la capacidad de acceder a una vida digna, en ese sentido, ha comentado que:

El trabajo identifica a las personas en su inserción comunitaria y con la propia idea del desarrollo humano moderno; el 
trabajo no solo define el lugar que ocupa en la estructura social sino también las relaciones sociales y la forma de afiliación social de los individuos. El trabajo decente no es únicamente una aspiración sino también la identificación plena del trabajo como derecho humano, tal como es definido internacionalmente. Los derechos inherentes al trabajo suponen un anhelo y un compromiso de todo el planeta para la plena realización de los hombres y mujeres (Organización Internacional del Trabajo, 2011, p. 12)ILO Office in Argentina, \& Confederaci $\backslash u c 0 \backslash \backslash u 243\{\} n$ General del Trabajo (Argentina.

Sin embargo, al contrastarlo con la realidad, las condiciones de trabajo de las empresas actualmente no logran alcanzar lo dictado por la OIT, así lo señala Prieto (2017) al indicar que el rasgo que mejor caracteriza esta crisis es el de la sustitución de la norma social del pleno empleo de la sociedad salarial por la del empleo flexible y precario de la sociedad postsalarial, idea que es compartida con Gálvez, Gutiérrez, Picazzo y Osorio (2016) al referir que el proceso globalizador llevó a que los trabajadores fueran perdiendo sus derechos laborales, ayudando a una mayor precarización, que es una de las causas del incremento en la desigualdad.

En este sentido, la situación actual donde comienza la hegemonía del trabajo precario es inherente a la globalización. De esta manera, el trabajo decente tiene un alcance universal, es un concepto que reconoce los derechos de las personas como seres humanos, no como miembros de uno u otro país; su planteamiento trasciende las fronteras nacionales (Sen, 2007) y se dirige a todo tipo de trabajadores: formales, informales y autónomos (Barreto, 2001). Por ello se orienta a impulsar una globalización más justa que ofrezca beneficios y oportunidades tangibles a todos los países y personas (Zubero, 2007 en Gálvez et al., 2016).
Frente a la globalización, surge la agenda de trabajo decente, que se enfoca no solo en tener trabajo para todos sino en garantizar que el trabajo sea de calidad y proveedor de mejores perspectivas de desarrollo personal y libertad de las personas para expresar sus preocupaciones, para organizar y participar en las decisiones que afectan sus vidas (ILO, 2013).

La necesidad de este enfoque es fundamental como respuesta a los modelos de precarización laboral cada vez más normalizados. Fue solo después de que la Cumbre Mundial sobre Desarrollo Social en 2005 adoptara el empleo y el trabajo decente como objetivo mundial, que el papel del trabajo decente en el desarrollo sostenible comenzó a recibir un reconocimiento constante (ILO, 2013).

Es así que el trabajo decente ha aparecido en las agendas internacionales y se ha relacionado con el desarrollo sostenible, tanto así, que el octavo de los 17 Objetivos del Desarrollo Sostenible (ODS) se refiere al trabajo decente y al crecimiento económico. El paradigma del trabajo decente es una propuesta y una necesidad para el combate de los procesos de precarización laboral, cada vez más acentuados y asociados a efectos adversos en la salud; y que, a su vez, van en detrimento del desarrollo sostenible.

\section{Trabajo precario}

Prieto (2017) indica que el rasgo que mejor caracteriza la crisis actual es el de la sustitución de la norma social del pleno empleo de la sociedad salarial por la del empleo flexible y precario de la sociedad postsalarial, idea que es compartida con Gálvez et al. (2016) al referir que el proceso globalizador llevó a que los trabajadores fueran perdiendo sus derechos laborales.

Prieto (2017) señala respecto a la definición hegemónica del trabajo/empleo en la sociedad salarial como tal, que lo más relevante de la etapa que se abre en los ochenta no es que 
haya desaparecido el pleno empleo, sino que, con el fin de facilitar la creación de empleo para hacer frente a una situación de paro masivo, se extendió la concepción del empleo "normal" hasta incluir casi cualquier tipo de empleo. De manera que un empleo asalariado temporal, a tiempo parcial o por cuenta propia (precario) sea tan normal - y, por lo tanto, tan políticamente "justo"- como un empleo por tiempo indefinido, a tiempo completo, estable, bien protegido socialmente y con perspectivas de mejora permanente.

El trabajo precario ha sido definido como la ausencia de los aspectos que caracterizan una relación de trabajo estándar (Rubery, Grimshaw, Keizer \& Johnson, 2018). Las empresas cada vez contratan más trabajadores que no reciben contrato estable con certidumbre laboral, beneficios y capacitación (Bidwell, 2009, Bidwell \& Briscoe, 2009). Las tendencias relacionadas incluyen menos oportunidades de ascenso, menor protección institucional como acceso a la salud y jubilación, dando como resultado, trabajo precario en el cual, existe una gran diferencia de poder entre los empleados y los empleadores. Aunque el trabajo precario no es nuevo, incrementó demasiado en los últimos años (Bidwell \& Mollick, 2015; Cobb, 2015; Kalleberg, 2009).

Por lo tanto, se ha indicado que el trabajo precario es un determinante social que afecta la salud de trabajadores, familias y comunidades. $\mathrm{Su}$ reciente popularidad ha sido encabezada por el aumento del empleo flexible; así como al incremento en el interés en variables sociales, incluidas la salud, las condiciones de trabajo y a la disponibilidad de nuevos sistemas de información (Benach, Vives, Amable, Vanroelen, Tarafa \& Muntaner, 2014).

Paralelamente al trabajo flexible han surgido los acuerdos del trabajo alternativo, los cuales, continúan creciendo en número y variedad. Se han identificado tres dimensiones que sustentan los arreglos del trabajo alternativo: a) flexibilidad en la relación laboral, b) flexibilidad en el horario de trabajo y c) flexibilidad en el lugar donde se realiza el trabajo. Así mismo, se identificaron dos caras en el nuevo mundo del trabajo, una, para los trabajadores altamente capacitados que escogen el trabajo alternativo y otra para aquellos trabajadores poco capacitados que luchan por ganarse la vida y dependen de las necesidades de la organización (Gretchen en Spreitzer, Cameron \& Garrett, 2017).

En ese sentido el trabajo alternativo suele ser precario; si bien, es una respuesta ante el desempleo, no llega a brindar la satisfacción, garantía y desarrollo personal que solo se obtiene a través del trabajo decente, más aún, encierra a los trabajadores en un círculo vicioso de temporalidad, bajos salarios, horarios incompatibles con la vida familiar y demás características que pueden traer tanto malestar como el desempleo.

\section{Impactos en la salud}

La disponibilidad de los nuevos sistemas de datos e información han aumentado la evidencia disponible sobre la asociación entre empleo precario y mala salud (Benach et al., 2014). La crisis económica global afecta la vida de las personas en diferentes esferas -social, laboral, individual y familiar-, sin embargo, de las secuelas más notables de dicha crisis son las concernientes al trabajo, como lo son: la precariedad laboral, la incertidumbre laboral, el desempleo, aunado a ello, la pobreza y marginación (Catalano, 1991). Otros autores que han referido efectos negativos a la salud por parte de la crisis económica y por ende de la precarización laboral, han sido Fountoulakis et al. (2014) y Sicras y Navarro-Artieda (2015), confirmando la relación de variables macroeconómicas con el suicidio y la depresión. México no es la excepción ante las problemáticas globales generadas por la crisis económica, además de ser un país en desarrollo que atraviesa grandes transiciones, especialmente a partir de los años setenta, con la incursión 
de la mujer en el mundo laboral, la transición hacia nuevos esquemas familiares, una mayor movilidad y concentración de la población rural a escenarios urbanos, cambios que auspiciaron un incremento del sector industrial en decremento del sector agrario, entre los cambios más relevantes (Mendoza \& Tapia, 2010).

En la misma línea la Organización Mundial de la Salud (OMS) (2011), manifiesta la importancia de estudiar la salud mental relacionada con los efectos de las crisis financieras, tales como deprivación, pobreza e inequidad, además señala a las crisis económicas como un factor de alto riesgo para la salud mental. Respecto al trabajo, postula que un ambiente laboral saludable y un estilo de vida sano son factores protectores determinantes en la salud mental, por el contrario, la pobreza, falta de educación, el desempleo, la incertidumbre y el estrés laboral son factores de alto riesgo para la salud mental. La puntuación en salud mental se deteriora progresivamente a medida que la puntuación en precariedad es más alta, además, la precariedad laboral se relaciona con un impacto negativo sobre el bienestar de los trabajadores y, por lo tanto, sobre la productividad de las organizaciones (Benach, Juliá, Tarafa, Mir, Molinero \& Vives, 2015).

La transición del trabajo permanente a otro estado laboral se asoció significativamente con los síntomas depresivos graves de nueva aparición (Sora, De Cuyper, Caballer, Peiró \& De Witte, 2013). Asimismo, el trabajo temporal también se asocia con angustia psicológica (Kawada, 2018), y el diseño del mismo es importante para el sentido de vida, la salud y el desarrollo de los individuos (Parker, 2014).

Además, otros indicadores subjetivos como la tensión se asocian a la interacción de un individuo con el ambiente de trabajo. Si las situaciones y eventos se evalúan como una amenaza (un factor estresante), surgen emociones negativas que pueden generar tensión psicológica, como ansiedad, tensión física, enfermedad cardíaca o tensión conductual, como fumar (Spector, 1998, en Parker 2014). A decir de Parker, el diseño del trabajo puede ser un poderoso vehículo para el aprendizaje y el desarrollo y para mantener y mejorar la salud física y mental de los empleados (Parker, 2014).

También se ha encontrado que largas horas de trabajo tienen consecuencias negativas para la salud, el bienestar familiar y el ocio (Jacobs \& Gerson, 2004, Kivimäki, 2015) y otros efectos como la fatiga y la reducción de la salud (Sánchez, 2017).

Asimismo, la literatura ha referido que los trabajadores con jornadas fuera de lo estándar se enfrentan a una gama de retos adicionales, desde la reducción del apoyo y la participación comunitaria, hasta problemas de salud e incremento de divorcios (Cornwell \& Warburton 2014, Davis et al., 2008, Kalil et al., 2010, PerryJenkins et al., 2007)

Estos mismos autores, señalan que las políticas respecto a la jornada laboral impactan en la salud y la felicidad de hombres y mujeres. Los efectos negativos en la parentalidad y en la felicidad desaparecen con la introducción de políticas respecto a la jornada laboral que permiten a los padres combinar su trabajo con las obligaciones familiares, por ejemplo, permisos pagados $\mathrm{y}$ días de vacaciones (Glass, Simon \& Andersson, 2016). Además, dejar las consecuencias en la salud de las condiciones laborales en último lugar solo perpetuará las desigualdades en salud por la ausencia de condiciones de trabajo decente.

Asimismo, respecto al trabajo decente, un trabajo con condiciones formales puede crear oportunidades significativas para la inclusión social y puede mejorar las condiciones de trabajo: seguridad, salud e ingresos (International Labour Organization, 2013). 
Estos hallazgos sugieren la relación entre trabajo decente y mayor salud, así como de trabajo precario y menor salud; lo que sustenta la idea central del presente estudio respecto a la asociación entre las condiciones de trabajo y la salud. En ese sentido, se convierte en una línea de investigación vital, como parte de una sociedad que transita del trabajo estándar hacia nuevos escenarios de trabajo. Estudiar esa transición, así como el análisis de los efectos del tipo de trabajo, además de plantear una base teórica, brinda argumentos para encontrar soluciones a beneficio de todos: empleados y empresas; ya que un individuo que goce de buena salud, además del derecho que tiene a esta y del bienestar que podrá gozar si la tiene, podrá aportar más en su familia, en la sociedad y por supuesto, mayor productividad en su lugar de trabajo.

Por último, a consideración de la ILO (2013), el trabajo decente incluirá beneficios como el acceso al cuidado de la salud, que se relacionará con mayor salud en los empleados, así mismo promueve que para lograr economías y medioambiente sostenibles se debe crear trabajo decente, mejorar la calidad de trabajos existentes y avanzar en la inclusión social.

\section{Trabajo y Objetivos del Desarrollo Sostenible}

El aspecto normativo del proyecto del desarrollo sostenible se orienta hacia cuatro objetivos definitorios de una buena sociedad: la prosperidad económica, la inclusión y la cohesión social, la sostenibilidad ambiental y la buena gobernanza por parte de los principales actores, entre ellos los gobiernos y las empresas (Sachs, 2015)2015. Asimismo señala que, hacer realidad el desarrollo sostenible en un planeta superpoblado, desigual y degradado, es el reto más importante al que se enfrenta la generación actual; por lo cual los Objetivos del Desarrollo Sostenible (ODS) deben ser la brújula para el desarrollo del planeta.
Los ODS, también conocidos como Objetivos Mundiales, son un llamado universal a la adopción de medidas para poner fin a la pobreza, proteger el planeta y garantizar que todas las personas gocen de paz y prosperidad. Estos 17 objetivos están interrelacionados, es decir, con frecuencia la clave del éxito de uno involucrará las cuestiones más frecuentemente vinculadas con otro (ONU, 2015).

Estos objetivos conllevan un espíritu de colaboración y pragmatismo para elegir las mejores opciones con el fin de mejorar la vida, de manera sostenible, para las generaciones futuras. Proporcionan orientaciones y metas claras para su adopción por todos los países en conformidad con sus propias prioridades y los desafíos ambientales del mundo en general. Abordan las causas fundamentales de la pobreza y nos unen para lograr un cambio positivo en beneficio de las personas y el planeta (ONU, 2015). En la tabla 1 se expone uno de los ODS y los objetivos específicos que más se relacionan con el tema abordado en estudio.

Tabla 1. Objetivo ocho del Desarrollo Sostenible (ONU, 2015)

\begin{tabular}{|c|c|}
\hline \multirow{3}{*}{$\begin{array}{l}\text { Objetivo } 8 . \\
\text { Promover el } \\
\text { crecimiento } \\
\text { económico } \\
\text { sostenido, } \\
\text { inclusivo y } \\
\text { sostenible, el } \\
\text { empleo pleno } \\
\text { y productivo } \\
\text { y el trabajo } \\
\text { decente para } \\
\text { todos }\end{array}$} & $\begin{array}{l}\text { 8.3 Promover políticas orientadas al } \\
\text { desarrollo que apoyen las actividades } \\
\text { productivas, la creación de empleo decente, el } \\
\text { emprendimiento, la creatividad y la innovación, } \\
\text { y alentar la oficialización y el crecimiento de } \\
\text { las microempresas y las pequeñas y medianas } \\
\text { empresas, entre otras cosas mediante el acceso } \\
\text { a servicios financieros }\end{array}$ \\
\hline & $\begin{array}{l}\text { 8.5 Para 2030, lograr el empleo pleno y } \\
\text { productivo y garantizar un trabajo decente para } \\
\text { todos los hombres y mujeres, incluidos los } \\
\text { jóvenes y las personas con discapacidad, y la } \\
\text { igualdad de remuneración por trabajo de igual } \\
\text { valor }\end{array}$ \\
\hline & $\begin{array}{l}\text { 8.8 Proteger los derechos laborales y promover } \\
\text { un entorno de trabajo seguro y protegido } \\
\text { para todos los trabajadores, incluidos los } \\
\text { trabajadores migrantes, en particular las } \\
\text { mujeres migrantes y las personas con empleos } \\
\text { precarios }\end{array}$ \\
\hline
\end{tabular}


Asistimos a un mundo con una gobernanza injusta. Las tendencias dominantes de nuestro tiempo han excluido del trabajo decente a millones de personas, las cuales se han visto obligadas a generar formas de sobrevivencia que los colocan en situación de absoluta desprotección e indignidad (Sánchez, 2014). Esta autora se pregunta si es sostenible un modelo económico donde el trabajador, sus derechos y su protección son la variable de ajuste para garantizar la competitividad y sobrevivencia según las leyes del mercado. En la globalización conviven estas tendencias con procesos que abogan por una globalización que llegó para quedarse, pero en la que es necesario acumular fuerza e influencia para impulsar maneras distintas de producir, distribuir, consumir, trabajar y tomar decisiones (Sánchez, 2014).

En este sentido, los objetivos del desarrollo sostenible constituyen una agenda de importancia vital en todos los ámbitos y deben ser la lupa sobre la que miremos y el borde en el que enmarquemos el fenómeno de la precariedad laboral, sabiendo que ya existen medidas y corrientes a nivel internacional que buscan permear sus efectos $\mathrm{y}$ marcan directrices en la búsqueda, tanto del trabajo decente, como de la promoción de la salud. El desarrollo sostenible al ser un modelo de desarrollo y una agenda internacional tripartita, económica, ambiental y social, abarca perfectamente las áreas que hasta ahora se han abordado en este estudio: trabajo y salud, de manera que deja denunciado al trabajo precario a los problemas de salud y busca soluciones al poner como meta al trabajo decente. Si bien son objetivos ambiciosos, y no son pocos los obstáculos que se oponen al logro del desarrollo sostenible en la práctica, es mucho más lo que se puede ganar (Sachs, 2015), y uno de los logros sociales es sin duda la salud pública.

\section{Conclusiones}

La revisión de estos estudios sugiere tres cosas: la importancia del trabajo decente, la problemática del trabajo precario y que, ambos, tienen impactos positivos $\mathrm{y}$ negativos en el individuo y, por lo tanto, en la sociedad. Resultados positivos son posibles, sin embargo, estos requieren políticas específicas que aprovechen las oportunidades y aborden los desafíos identificados mediante la integración de los elementos sociales y de trabajo decente y garanticen una transición suave y justa hacia economías sostenibles. De hecho, las oportunidades de ganancias pueden ser mayores en los países en desarrollo y las economías emergentes (International Labour Organization, 2013).

Mejorando directamente la paga y las condiciones de trabajo en los grupos con mayor riesgo de precariedad y, al mismo tiempo, siendo ejemplo para que consejos locales y regionales continúen con estrategias similares para ser socialmente responsables, las empresas avanzan en su camino a serlo, sin embargo la adquisición de este título requiere más que la fijación de normas en papel; se requieren esfuerzos significativos de todas las partes para incorporar condiciones de trabajo dignas (Jaehrling, Johnson, Larsen, Refslund \& Grimshaw, 2018)

De esta manera, y siguiendo las ideas de estos autores, las políticas que encaminen sus esfuerzos a mejorar las condiciones laborales, y a acceder al trabajo decente, son necesarias para lograr combatir la precariedad laboral y junto con ella, sus impactos negativos en la salud de los individuos, trayendo con esto mejoras multidimensionales: individuales, familiares, sociales y empresariales. Por lo tanto, abonando al desarrollo sostenible, a nivel local, regional e internacional. El trabajo pues, es central para el desarrollo humano y sostenible y su estudio, desde todas las perspectivas posibles, permitirá señalar la pauta que debe seguirse para que el trabajo sea en efecto, impulsor de desarrollo y no un generador de mayores problemas sociales que comprometan a la salud. 
Así pues, el trabajo precario queda expuesto como problemática de primer orden, mientras que el trabajo decente es un medio y objetivo para el desarrollo sostenible, teniendo como base importante el objetivo número ocho de los ODS.

\section{Referencias}

Barreto, H. (2001). Concepto y dimensiones del trabajo decente: entre la protección social básica y la participación de los trabajadores en la empresa. Gaceta Laboral, 7(2), 181199.

Benach, J., Julià, M., Tarafa, G., Mir, J., Molinero, E. \& Vives, A. (2015). La precariedad laboral medida de forma multidimensional: distribución social y asociación con la salud en Cataluña. Gaceta Sanitaria, 29(5), 375-378. https://doi.org/10.1016/j. gaceta.2015.04.002

Benach, J., Vives, A., Amable, M., Vanroelen, C., Tarafa, G. \& Muntaner, C. (2014). Precarious Employment: Understanding an Emerging Social Determinant of Health. Annual Review of Public Health, 35(1), 229-253. https://doi.org/10.1146/ annurev-publhealth-032013-182500

Bidwell, M. (2009). Do peripheral workers do peripheral work? Industrial and Labor Relations Review, 62(2), 200-25

Bidwell, M. \& Briscoe, F. (2009). Who contracts? Determinants of the decision to work as an independent contractor among information technology workers. Academy of Management Journal, 52(6), 1148-68

Bidwell, M. \& Mollick, E. (2015). Shifts and ladders: comparing the role of internal and external mobility in managerial careers. Organization Science, 26(6), 1629-45
Economic Insecurity. American Journal of Public Health, 81(9), 1148-1152. https:// doi.org/10.2105/ajph.81.9.1148

Cobb, J.A. (2015). Risky business: the decline of defined benefit pensions and firms' shifting of retirement risk. Organization Science, 26(5), 1332-50

Cornwell, B. \& Warburton, E. (2014). Work schedules and community ties. Work and Occupations, 41, 139-74

Davis, K. D., Benjamin Goodman, W., Pirretti, A. E., \& Almeida, D. M. (2008). Nonstandard Work Schedules, Perceived Family Well-Being, and Daily Stressors. Journal of Marriage and Family, 70(4), 991-1003. doi:10.1111/j.17413737.2008.00541.x

Fountoulakis, K. N., Kawohl, W., Theodorakis, P. N., Kerkhof, A. J. F. M., Navickas, A., Hoschl, C., ... Lopez-Ibor, J. (2014). Relationship of suicide rates to economic variables in Europe: 2000-2011. The British Journal of Psychiatry, 205(6), 486-496. https://doi.org/10.1192/bjp. bp.114.147454

Gálvez, E., Gutiérrez, E., Picazzo, E. \& Osorio, J. (2016). El trabajo decente, una alternativa para reducir la desigualdad en la globalización: el caso de México. Región y sociedad, 28(66), 55-94.

Glass, J., Simon, R. W., \& Andersson, M. A. (2016). Parenthood and Happiness: Effects of Work-Family Reconciliation Policies in 22 OECD Countries. American Journal of Sociology, 122(3), 886929. doi:10.1086/688892

International Labour Organization. (2013). Building a future with decent work. 102nd Session of the International Labour Conference. Geneva.

Catalano, R. (1991). The Health Effects of 
Jacobs, J. \& Gerson, K. (2004). The Time Divide: Work, Family and Gender Inequality. Cambridge, MA: Harvard Univ. Pres

Jaehrling, K., Johnson, M., Larsen, T. P., Refslund, B. \& Grimshaw, D. (2018). Tackling precarious work in public supply chains: A comparison of local government procurement policies in Denmark, Germany and the UK. Work, Employment and Society, 32(3), 546-563.

Kalil, A., Ziol-Guest, K. M., \& Levin Epstein, J. (2010). Nonstandard Work and Marital Instability: Evidence From the National Longitudinal Survey of Youth. Journal of Marriage and Family, 72(5), 1289-1300. doi:10.1111/j.17413737.2010.00765.x

Kalleberg, A. (2009). Precarious work, insecure workers: employment relations in transition. American Sociological Review, 74(1), 1-22

Kawada, T. (2018). Temporary work and depressive symptoms. Occupational Medicine, 68(4), 290-290. https://doi. org/10.1093/occmed/kqy048

Kivimäki, M. (2015). Long working hours and risk of coronary heart disease and stroke: a systematic review and meta-analysis of published and unpublished data for 603 838 individual. Lancet 386,1739-46

Mendoza, M. E. \& Tapia, G. (2010). Situación Demográfica de México 1910-2010. En Situación Demográfica de México 19102010 (CONAPO, pp. 1-14). México.

Organización Internacional del Trabajo, (OIT). (1999). Memoria del director general: Trabajo decente. 87a. Reunión de la Conferencia Internacional del Trabajo. Junio. Ginebra, Cantón de Ginebra.
Organización Internacional del Trabajo, (OIT). (2011). Trabajo decente para una vida digna. Argentina.

Organización Mundial de la Salud, (OMS). (2011). Impact of economic crises on mental health. Denmark: Organización Mundial de las Naciones Unidas (ONU). Recuperado a partir de http://www.euro. who.int/en/home

Organización de las Naciones Unidas, (ONU) (2015). La Asamblea General adopta la Agenda 2030 para el Desarrollo Sostenible. Recuperado a partir de http:// bit.ly/2qTfs $8 n$

Parker, S. K. (2014). Beyond Motivation: Job and Work Design for Development, Health, Ambidexterity, and More. Annual Review of Psychology, 65(1), 661691. https://doi.org/10.1146/annurevpsych-010213-115208

Perry-Jenkins, M., Goldberg, A. E., Pierce, C. P., \& Sayer, A. G. (2007). Shift Work, Role Overload, and the Transition to Parenthood. Journal of Marriage and Family, 69(1), 123-138. doi:10.1111/ j.1741-3737.2006.00349.x

Prieto, C. (2017). El futuro del trabajo (decente): de la hegemonía a su crisis, de la centralidad exclusiva a una centralidad compartida (con los ciudadanos). Futuro del Trabajo: Trabajo decente para todos, (3), 8-9.

Rubery, J., Grimshaw, D., Keizer, A. \& Johnson, M. (2018). Challenges and contradictions in the "normalising" of precarious work. Work, Employment and Society, 32(3), 509-527.

Sachs, J. (2015). La era del desarrollo sostenible (Planeta libros). España: Planeta libros. 
Sánchez, R. (2017). Does a mandatory reduction of standard working hours improve employees' health status? Industrial Relations, 56(1), 3-39

Sánchez Ferrer, Y. (2014). Trabajo decente y desarrollo sostenible. Para bailar se necesitan dos. Cayapa. Revista Venezolana de Economía Social, 14(28).

Sen, A. (2007). El trabajo decente, un derecho humano. En A. Sen, J. Stiglitz, \& I. Zubero, Se busca trabajo decente (pp. 121-133). Madrid: Ediciones hoac.
Sicras, A. \& Navarro-Artieda, R. (2015). Use of antidepressants in the treatment of major depressive disorder in primary care during a period of economic crisis. Neuropsychiatric Disease and Treatment, 29. https://doi.org/10.2147/NDT.S91227

Sora, B., De Cuyper, N., Caballer, A., Peiró, J. M. \& De Witte, H. (2013). Outcomes of Job Insecurity Climate: The Role of Climate Strength. Applied Psychology, 62(3), 382-405. https://doi.org/10.1111/j.14640597.2012.00485.x 